\title{
Surface transfer doping can mediate both colloidal stability and self-assembly of nanodiamonds
}

Tristan Petit ${ }^{a}$, Hugues A. Girard ${ }^{a}$, Adeline Trouvéa, Isabelle Batonneau-Gener ${ }^{b}$, Philippe Bergonzo ${ }^{a}$; Jean-Charles Arnaulta

a CEA, LIST, Diamond Sensors Laboratory, F-91191 Gif-sur-Yvette, France E-mail: jean-charles.arnault@cea.fr

${ }^{\mathrm{b}}$ Institut de Chimie des Milieux et Matériaux de Poitiers, CNRS-UMR 7285, 4 rue Michel Brunet, B27, F-86022 Poitiers cedex, France

\begin{abstract}
Although undoped diamond is insulating, hydrogenated bulk diamond surfaces exhibit surface conductivity under air and are electrochemically active in aqueous solutions. Due to their large surface/volume ratio, similar surface effects may exhibit a dramatic impact on the properties of nanodiamonds. Here we show that plasma-hydrogenated detonation nanodiamonds (NDs$\mathrm{H}$ ) display a positive zeta potential in water due to charge transfer with a redox couple involving oxygen in water. The transfer doping of NDs-H in water can be modulated by $\mathrm{pH}$. Surprisingly, after acid addition, strong Coulomb coupling between NDs-H and adsorbed counterions induces the self-assembly of NDs-H into organized macro-structures reaching millimeter scale.
\end{abstract}
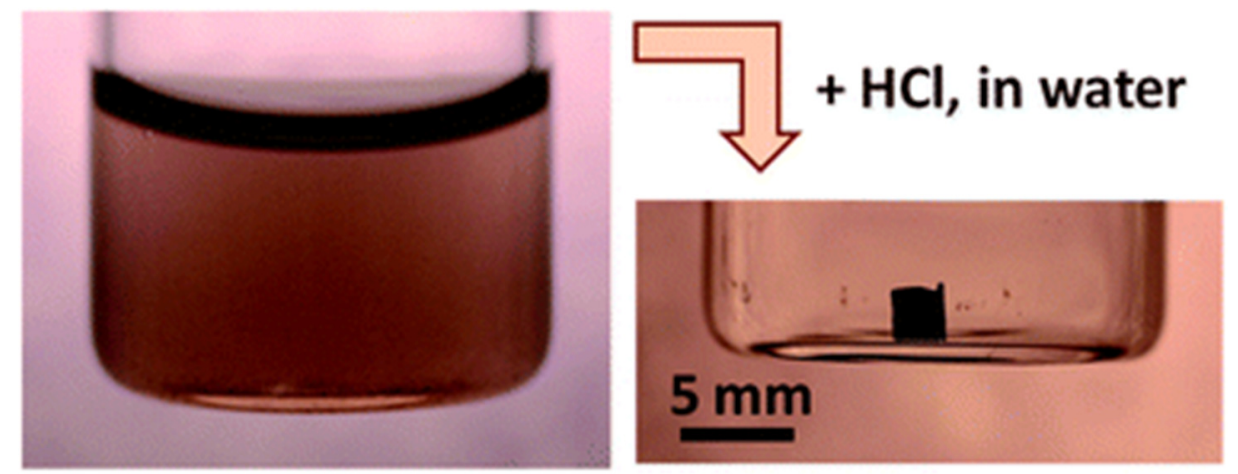

Nanomaterials often exhibit fundamentally new properties when compared to their bulk counterparts. Quantum confinement effects, high surface/volume ratio or new material crystal phases are some of the reasons for these new properties. ${ }^{1}$ In the case of nanodiamonds (NDs) produced by detonation synthesis, ${ }^{2}$ astonishing properties such as exceptional colloidal stability, ${ }^{3,4}$ giant permittivity ${ }^{5}$ or unusual charge exchange properties ${ }^{6}$ are hardly explained by the properties of their diamond core only. In contrast, peculiar surface phenomena observed on hydrogenated bulk diamond material have not been reported for NDs yet, such as the surface transfer doping occurring after air exposure ${ }^{7,8}$ or in water ${ }^{9}$ and their unique chemical reactivity. ${ }^{10,11}$ Similar surface effects may strongly influence ND properties due to their large surface to volume ratio. Here we show that surface transfer doping exists for NDs, and, beyond an electrostatic stabilization of the hydrogenated NDs (NDs-H) in water (zeta potential in water is $+40 \mathrm{mV}$ ), it also mediates their self-assembly into millimeter scale structures in solution when brought to acidic $\mathrm{pH}$ values. NDs of larger size, synthesized by the High Pressure High Temperature (HPHT) method, could not be dispersed in water after similar hydrogenation treatment therefore the effects related here are specific to detonation NDs-H. NDs are generally considered as inert nanoparticles however our result demonstrates that surface 
treatments such as hydrogenation can enhance electronic properties of the nanodiamond core. Driving NDs-H electrostatic interactions by modulation of their electrochemical environment may open new perspectives for self-assembly and also for gene delivery.

NDs-H were prepared by microwave-enhanced plasma treatments as described elsewhere. ${ }^{12}$ Hydrogen plasma treatments have been shown to be the most adapted method to efficiently passivate the ND surface with hydrogen atoms while etching non-diamond carbon and oxygen species. ${ }^{13}$ In a previous study, we demonstrated that vanishing of the oxygenated terminations and the presence of $\mathrm{C}-\mathrm{H}$ bonds after such plasma treatments occur, evidenced by FTIR and XPS analysis. ${ }^{12}$ Furthermore, hydrogenation of NDs was also confirmed by the validation on our NDs-H of grafting routes specific to hydrogen-terminated diamond surfaces. ${ }^{10,14,15}$

First, interactions between NDs and water molecules were investigated. The colloidal stability of NDs-H is unexpected since hydrogen-terminated bulk diamond surfaces are known to be hydrophobic. ${ }^{16}$ Water adsorption isotherms on NDs-H and oxidized NDs (NDs-COOH), used as a reference for hydrophilic NDs, are plotted and shown in Fig. 1. Surprisingly, a stronger water adsorption is observed on NDs-H compared to NDs-COOH. By taking into account their specific surface area, the density of hydrophilic sites can be estimated to be 1.4 and 1.7 sites per $\mathrm{nm}^{2}$ on $\mathrm{NDs}-\mathrm{COOH}$ and $\mathrm{NDs}-\mathrm{H}$, respectively (see ESI + ). ${ }^{17}$ Water molecules interact strongly with the NDs-H surface as shown by the large mass increase at low relative water pressure, unlike with NDs- $\mathrm{COOH}\left(P / P_{0}<0.05\right.$, inset of Fig. $\left.1 \mathrm{~A}\right)$. In this pressure range, the mass increase is most probably related to electrostatic or hydrogen bonding of water molecules as well as water dissociation on the NDs-H surface. Water dissociation has already been observed on polycrystalline diamond and leads to the formation of hydroxyl groups. ${ }^{18}$ These hydrophilic sites on NDs-H are likely to facilitate the dispersion of NDs, initially thought to be hydrophobic, in water.

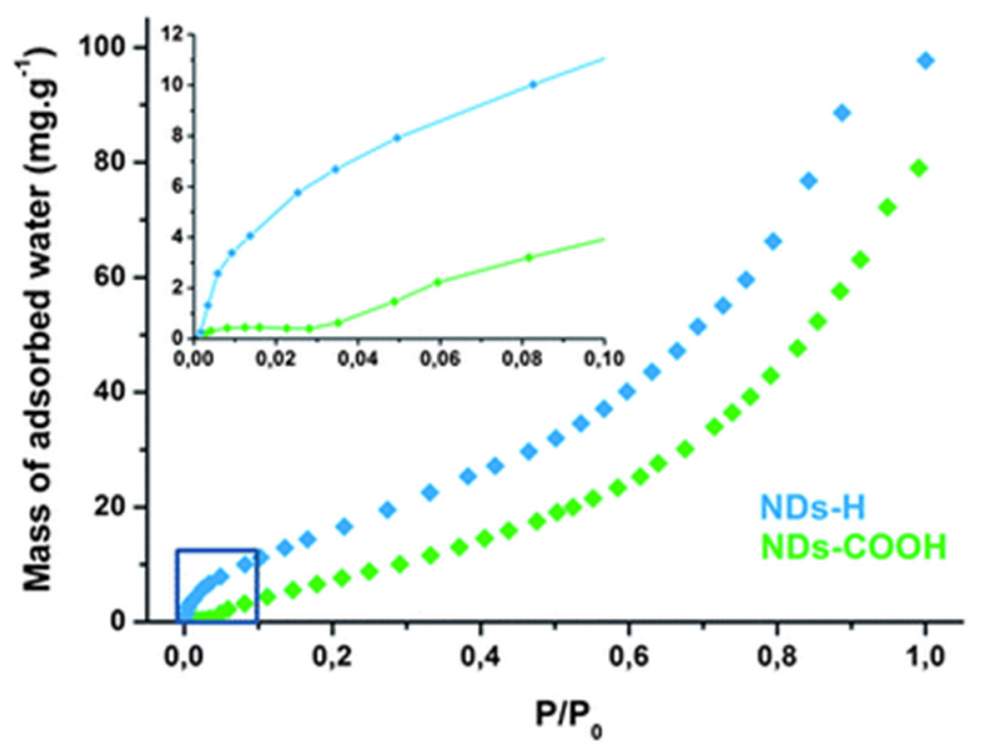

Fig. 1 Water adsorption on surface-modified nanodiamonds. Water vapor adsorption isotherms on NDs-H (blue) and NDs-COOH (green). Prior to water vapor exposure, the surface of NDs was annealed at $300^{\circ} \mathrm{C}$ under vacuum to remove physisorbed and weakly bound species. The inset shows a magnified view of adsorption at low water relative pressure $\left(P / P_{0}<0.1\right)$.

† Electronic supplementary information (ESI) available: Experimental procedures, specific surface area and hydrophilic site density calculations and the assembly experiment using sulfuric acid. See DOI: 10.1039/c3nr02492j 
Colloidal properties of NDs-H were then evaluated. After $2 \mathrm{~h}$ ultrasonication in water, stable suspensions of NDs-H are formed (Fig. S2 and S3t). NDs-H display a highly positive ZP, stabilized around a value of $+45-50 \mathrm{mV}$ for $\mathrm{pH}$ values in the range of 4 to 9 (Fig. 2A). From pH $=9$, the ZP decreases progressively up to an isoelectrical point (IEP), i.e. the point of zero charge, at $\mathrm{pH}=12.4$. Chakrapani et al. have shown that in water, the hydrogenated diamond surface can exchange charges with an electrochemical redox couple involving oxygen. ${ }^{9} \mathrm{O}$ diamond microparticles, the authors demonstrated that hole accumulation on the diamond surface leads to a positive $\mathrm{ZP}$ at $\mathrm{pH}$ below 6 . Considering that the positive $\mathrm{ZP}$ has the same origin on $\mathrm{NDs}-\mathrm{H}$, with the acidic character coming from atmospheric $\mathrm{CO}_{2}$, the following reaction takes place on the NDs-H surface:

$4\left(\mathrm{e}^{-} \mathrm{h}^{+}\right)_{\mathrm{NDs}-\mathrm{H}}+\mathrm{O}_{2, \mathrm{air}}+2 \mathrm{H}_{2} \mathrm{O}+4 \mathrm{CO}_{2, \mathrm{air}} \rightleftharpoons 4 \mathrm{~h}_{\mathrm{NDs}-\mathrm{H}^{+}}+4 \mathrm{HCO}_{3, \mathrm{aq}^{-}}$
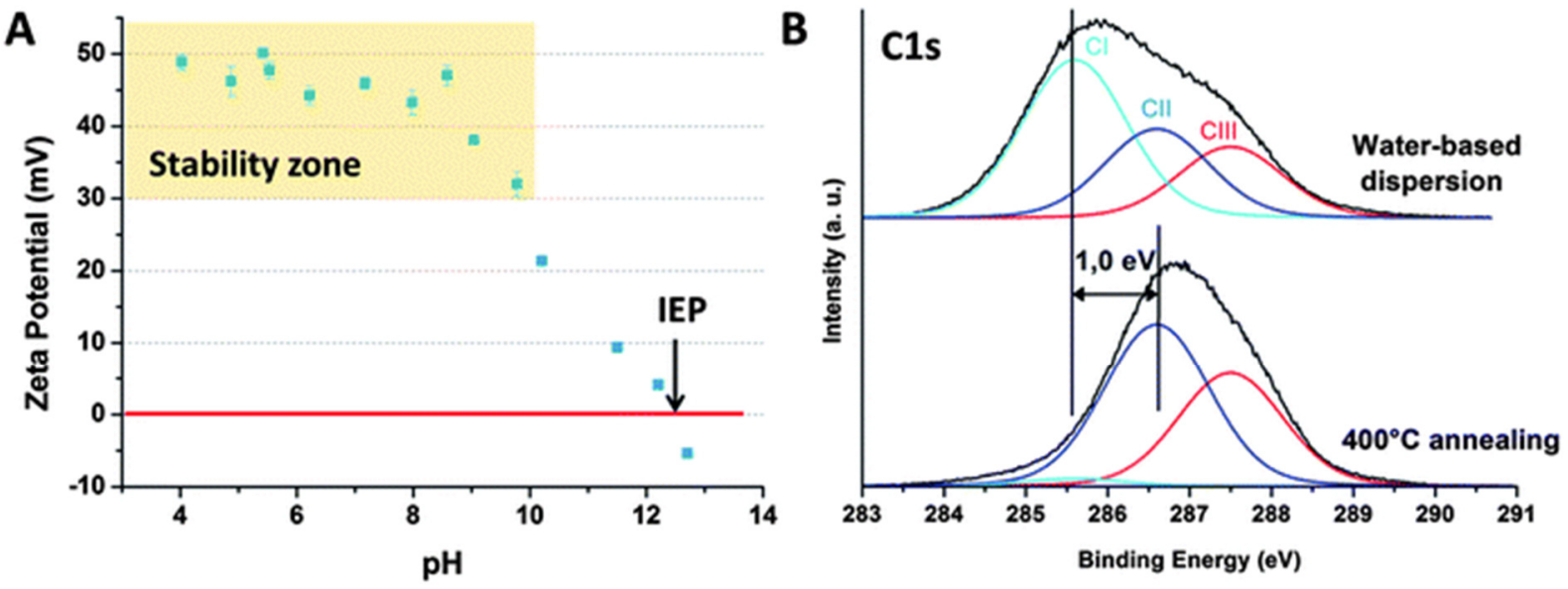

C

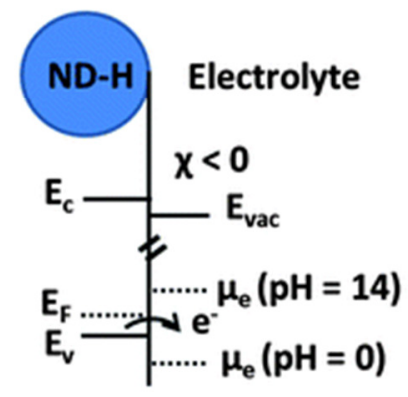

Before equilibrium

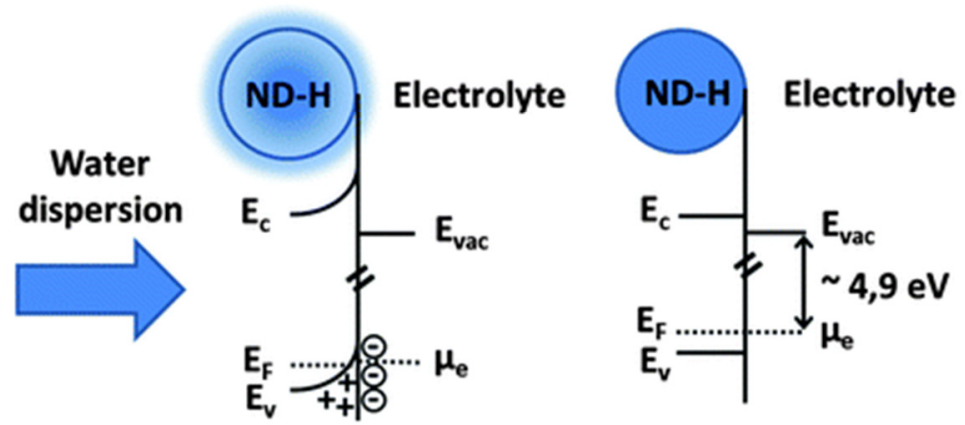

Positive Zeta potential Isoelectrical point

Fig. 2 Surface transfer doping on NDs-H in water. (A) Evolution of the Zeta potential of NDs-H in water. (B) Evolution of the XPS C1s spectrum of NDs-H after dispersion in water (top) and after in situ annealing at $400{ }^{\circ} \mathrm{C}$ (bottom). (C) Schematic view of the transfer doping model on $\mathrm{NDs}-\mathrm{H}$ in water. The electron density in and around the ND-H is illustrated in blue color.

In this model, the charge transfer direction depends on the equilibrium between the electrochemical potential of the oxygen redox couple, driven by $\mathrm{pH}$, and the Fermi level of the $\mathrm{NDs}-\mathrm{H}$. The key role of oxygen in the electrochemical process was validated by flushing the solution with $\mathrm{N}_{2}$, which resulted in a rapid flocculation of the NDs-H, probably related to the loss of positive charges on their surface. The IEP thus corresponds to the $\mathrm{pH}$ at which, out of the equilibrium, the electrochemical potential of the redox couple equals to the Fermi level of the NDs-H. Thus, when the equilibrium is reached, no band bending occurs, and there is no accumulation layer. A schematic band model of transfer doping on NDs-H in water is proposed in Fig. 2C. By using the Nernst equation and considering a temperature of $298 \mathrm{~K}$ and a partial pressure of $\mathrm{O}_{2}$ of 0.21 bar, an electrochemical potential of $-4.9 \mathrm{eV}$ is estimated for $\mathrm{pH}_{\text {IEP }}=$ 
12.4. This value is higher than the one obtained on microparticles $(-5.3 \mathrm{eV})$ by Chakrapani et al., which may be related to nanosize effects. As a consequence, NDs-H exhibit a large band bending, leading to a high $\mathrm{ZP}$ up to $\mathrm{pH}=9$, which is not the case for microparticles.

The presence of a hole accumulation layer on the NDs-H after air exposure and water dispersion was validated by X-ray Photoelectron Spectroscopy (XPS). Oxygen and nitrogen atomic contents extracted from the $01 \mathrm{~s}$ and N1s peaks are estimated as 3.0 and 5.7 at\%, respectively. In situ experiments have previously shown that oxygen is fully desorbed after hydrogen plasma treatment under similar conditions. ${ }^{13}$ Therefore detected oxygen mostly results from adsorption of oxygenated molecules, which is in agreement with water adsorption measurements (Fig. 1). Note that this point is also in agreement with previous FTIR analysis of such hydrogenated NDs, which revealed the necessity to anneal the samples under vacuum to desorb these oxygen-related species from their surface. ${ }^{12}$ The $\mathrm{C} 1 \mathrm{~s}$ spectrum of NDs-H was fitted with 3 components $\mathrm{Cl}$, CII and CIII with full widths at half-maximum (FWHM) of $1.5 \mathrm{eV}$ at 285.6, 286.6 and $287.5 \mathrm{eV}$, respectively (Fig. 2B). Attributions of these different components are difficult at this stage, because adsorbates influence the XPS signature of the NDs-H. Indeed, adsorbates are known to induce the formation of a surface conductive layer on hydrogenated bulk diamond by transfer doping. ${ }^{8}$ As a consequence, downshifts of $0.7-0.8 \mathrm{eV}$ of the $\mathrm{C} 1 \mathrm{~s}$ core level of hydrogenated bulk diamond have already been reported after air exposure, ${ }^{19}$ or adsorption of electron acceptors. ${ }^{20,21} \mathrm{An}$ in situannealing at $400{ }^{\circ} \mathrm{C}$ was thus performed to remove adsorbed moieties followed by a second XPS analysis. Surprisingly, the $\mathrm{Cl}$ component almost disappears after adsorbate removal (from 50 to $3 \%$ of the total carbon area after $2 \mathrm{~h}$ annealing at $400{ }^{\circ} \mathrm{C}$ ), while the oxygen content drops to $1.0 \mathrm{at} \%$. The two other components $\mathrm{Cll}$ and $\mathrm{CIII}$ are not affected by the annealing treatment. CII is thus attributed to undoped $\mathrm{sp}^{3}$ carbon bonds from the diamond core. CIII is mostly related to structural defects and $\mathrm{C}-\mathrm{N}$ bonds as discussed elsewhere. ${ }^{22,23} \mathrm{CH}_{x}(x=1$ to 3$)$ bonds induced by the hydrogen plasma treatment may also contribute to the CIII component. ${ }^{24}$ The binding energy difference between $\mathrm{Cl}$ and $\mathrm{Cll}$ is of $1.0 \mathrm{eV}$, which is comparable to the $\mathrm{C} 1 \mathrm{~s}$ downshift observed on bulk diamond after transfer doping. The $\mathrm{Cl}$ component is therefore related to a surface conductive layer on the NDs-H, induced by transfer doping after air exposure and dispersion in water. Considering that the whole ND volume is probed by XPS, transfer doping thus affects half of the carbon atoms in the NDs-H.

Note that for surface-graphitized NDs, a totally different XPS C1s peak evolution after annealing has been observed. ${ }^{22}$ Transfer doping is therefore specific to NDs-H and the positive ZP of surface-graphitized NDs has another origin which has been discussed elsewhere. ${ }^{22}$

Since transfer doping on diamond depends on the $\mathrm{pH}$ in water, it can be increased by acid addition. After addition of $\mathrm{HCl}$, the presence of $\mathrm{Cl}^{-}$counterions in the solution should be considered and the electrochemical equilibrium at the NDs-H surface described in eqn (1) is replaced by:

$4\left(\mathrm{e}^{-} \mathrm{h}^{+}\right)_{\mathrm{NDs}-\mathrm{H}}+\mathrm{O}_{2, \text { air }}+4 \mathrm{HCl} \rightleftharpoons 4 \mathrm{~h}_{\mathrm{NDs}-\mathrm{H}^{+}}+4 \mathrm{Cl}_{\mathrm{aq}}{ }^{-}+2 \mathrm{H}_{2} \mathrm{O}$

The addition of $\mathrm{HCl}$ results in an increase of the hole density on the NDs-H surface, which is compensated by $\mathrm{Cl}^{-}$counterions adsorption. When equilibrium (2) dominates, addition of $\mathrm{HCl}$ should not result in a pH decrease since protons are directly converted into holes in the NDs$\mathrm{H}$ and the only end-product would be water. In Fig. 3, the ZP of NDs- $\mathrm{H}$ is plotted after $\mathrm{HCl}$ addition to a water-based dispersion containing $6 \mathrm{ml}$ of NDs-H at a concentration of $0.8 \mathrm{mg}$ $\mathrm{ml}^{-1}$. The initial $\mathrm{pH}$ of the NDs-H dispersion is slightly acidic (5.9) due to the dissolved $\mathrm{CO}_{2}$ as discussed earlier. Although the surface charge of NDs-H increases upon acid addition, the ZP progressively decreases. ZP depends not only on the surface charge of NDs-H but also on the counterions in the Helmholtz diffuse layer surrounding the $\mathrm{NDs}-\mathrm{H},{ }^{25}$ therefore the strong 
adsorption of $\mathrm{Cl}^{-}$counterions on the NDs-H surface reduces the $\mathrm{ZP}$. For a $\mathrm{HCl}$ concentration of $20 \mathrm{mM}$, the measured $\mathrm{pH}$ is 3.7 while it should be 1.7 considering the proton concentration related to $\mathrm{HCl}$ addition. Therefore $\mathrm{NDs}-\mathrm{H}$ behave like proton sponges at acidic $\mathrm{pH}$, which is in agreement with the transfer doping model. For $20 \mathrm{mM} \mathrm{HCl}$, the hole density in the NDs-H is estimated to be $10^{17}$ to $10^{18}$ holes per $\mathrm{cm}^{3}$ considering that the whole NDs-H volume is doped (which corresponds to underestimation of the hole density since only the surface volume should be doped according to XPS measurements).

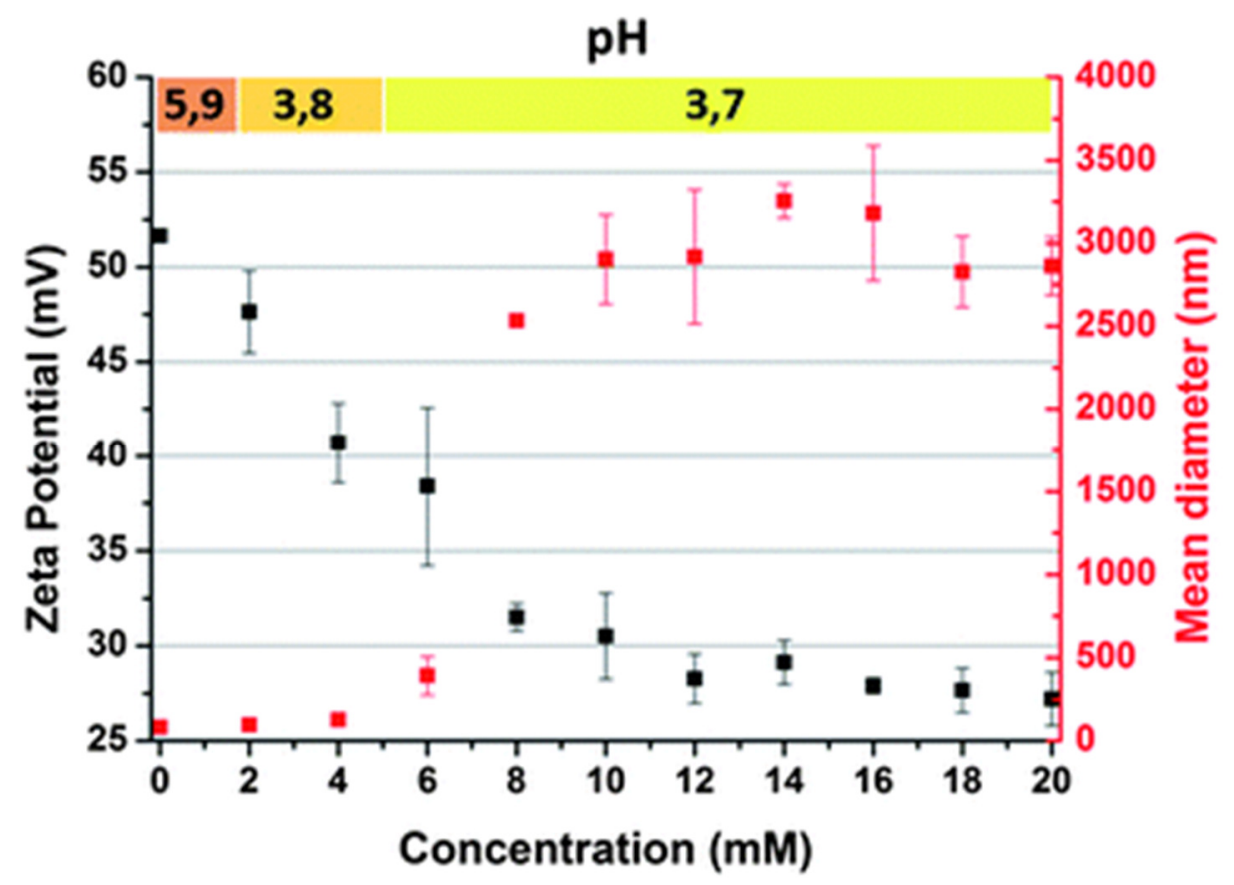

Fig. 3 Effects of acid addition in a water-based dispersion of NDs-H. The Zeta potential (black) and mean diameter of $\mathrm{NDs}-\mathrm{H} / \mathrm{Cl}^{-}$aggregates (red) are plotted after $\mathrm{HCl}$ addition. The initial $\mathrm{NDs}-\mathrm{H}$ concentration is $0.8 \mathrm{mg} \mathrm{ml}^{-1}$. $\mathrm{pH}$ values measured at each point are indicated on top.

In parallel to hole accumulation, the size increase of NDs-H aggregates is observed (Fig. 3). Up to $6 \mathrm{mM} \mathrm{HCl}$, the size increase is limited below $500 \mathrm{~nm}$ while a sharp increase up to the micrometer scale is measured at $8 \mathrm{mM}$. The aggregation cannot be attributed to a low colloidal stability since the ZP of $\mathrm{NDs}-\mathrm{H}$ is still higher than $+30 \mathrm{mV}$, normally ensuring a good electrostatic stabilization. Aggregation is most probably related to electrostatic interactions between $\mathrm{Cl}^{-}$counterions and $\mathrm{NDs}-\mathrm{H}$. Allahyarov et al. have shown that strong Coulomb coupling between nanoparticles and counterions can induce an attractive force between likecharged nanoparticles through a Coulomb depletion effect ${ }^{26}$ Briefly, for large densities of counterions adsorbed on nanoparticles by a strong Coulomb coupling, repulsive forces between counterions dominate over repulsive forces between the nanoparticles and create a depletion zone between two nearby nanoparticles. This Coulomb depletion results in a counterion-induced attractive force between the two like-charged nanoparticles, leading to aggregation. Here, Coulomb depletion forces are likely to appear at high $\mathrm{HCl}$ concentrations due to the high surface charge of NDs-H and the strong Coulomb coupling between NDs-H and $\mathrm{Cl}^{-}$counterions.

Aggregates formed by $\mathrm{HCl}$ addition where observed by Scanning Electron Microscopy (SEM) (Fig. 4A-C). At low concentrations, aggregates have spherical shapes (Fig. 4A). Surprisingly, cubic structures with smooth surfaces appear at higher concentrations (Fig. 4B and C), with a high reproducibility on several samples. Organized structures with sizes ranging up to the millimeter scale resulting from $\mathrm{NDs}-\mathrm{H} / \mathrm{Cl}^{-}$ion coupling were even observed in solution at a $\mathrm{HCl}$ 
concentration of $80 \mathrm{mM}$ (Fig. 4D). Similar experiments performed with NDs-COOH did not result in any organized structure therefore transfer doping certainly plays an important role in the self-assembly process. Other reports suggested that Coulomb interactions between facets of detonation NDs, presenting different electrostatic potentials, may impact their selfassembly. ${ }^{27,28}$ Although few HRTEM investigations reported facets on detonation NDs, ${ }^{29}$ their presence on our NDs-H has not been demonstrated.
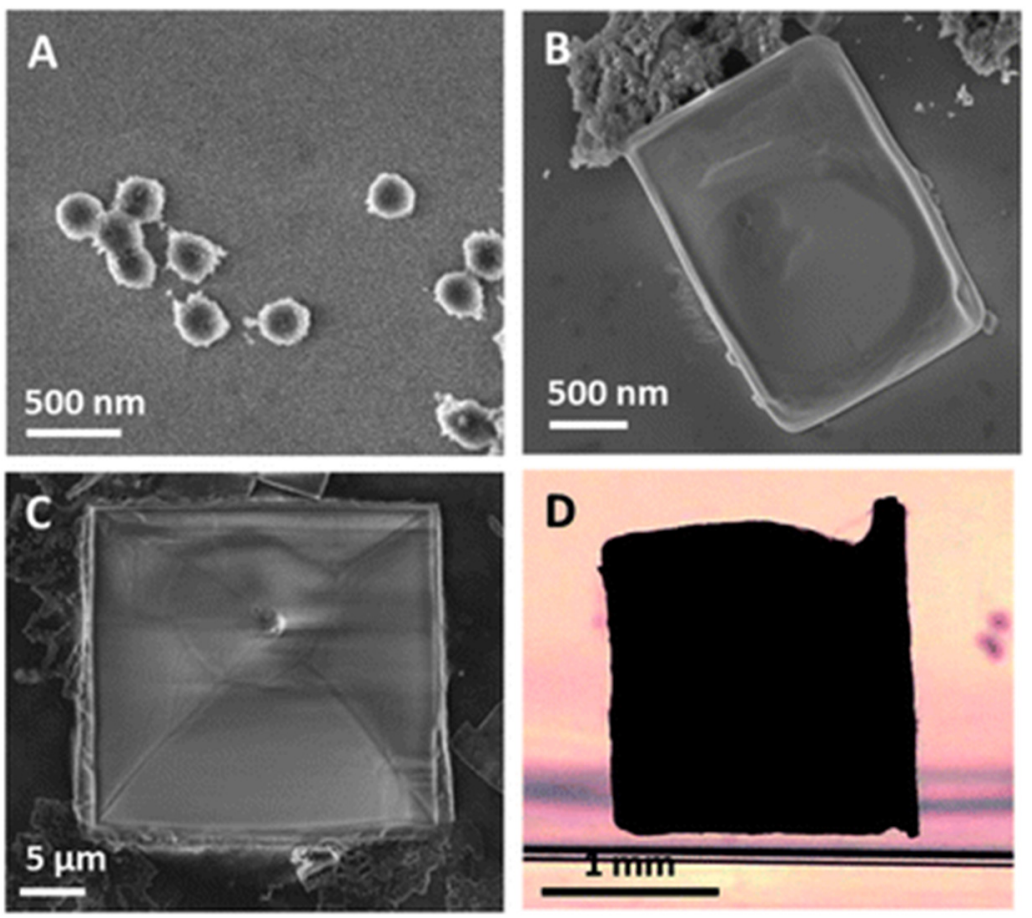

Fig. 4 Self-assembled NDs-H/Cl' ${ }^{-}$complexes. (A-C) SEM pictures of self-assembled structures for increasing $\mathrm{HCl}$ concentrations. (D) Optical image of a self-assembled structure with dimensions $1.7 \times 1.7 \times 0.8 \mathrm{~mm}^{3}$.

Furthermore, the valence of counterions in water also plays a critical role in the shape formation of the self-assembled structures. Indeed, similar experiments were performed with sulfuric acid $\left(\mathrm{H}_{2} \mathrm{SO}_{4}\right)$, resulting in the adsorption of $\mathrm{SO}_{4}{ }^{2-}$ counterions (see $\mathrm{ESI} \dagger$ ). In this case, large scale self-assembly was also observed at lower acid concentrations and resulted in rodlike structures (Fig. S4T). The effect of the counterions on the shape of the macrostructures is an intriguing question which remains to be explained.

To conclude, we anticipate that self-assembly induced by transfer doping may be applied to other nanomaterials based on graphene and organic semiconductors, ${ }^{30}$ silicon $^{31}$ or other semiconducting materials ${ }^{32}$ which are also sensitive to transfer doping. By using various acids and electron acceptors, more complex shapes might be obtained directly in solution. The proton sponge effect observed on NDs-H at acidic $\mathrm{pH}$ also suggests that they are good candidates as vectors for gene delivery since cationic nanoparticles with buffering properties already showed enhanced gene transfection capabilities. ${ }^{33}$ 


\section{Notes and references}

1. G. Hodes Adv. Mater., 2007, 19 , 639 -655.

2. V. Mochalin, O. Shenderova, D. Ho and Y. Gogotsi , Nat. Nanotechnol., 2012, 7,11 -23 .

3. M. Ozawa, M. Inaguma, M. Takahashi , F. Kataoka , A. Krüger and E. Ōsawa , Adv. Mater., 2007, 19 , $1201-1206$.

4. O. A. Williams, J. Hees, C. Dieker, W. Jäger, L. Kirste and C. E. Nebel , ACS Nano, 2010, $4,4824-4830$.

5. S. S. Batsanov , S. M. Gavrilkin , A. S. Batsanov, K. B. Poyarkov , I. I. Kulakova , D. W. Johnson and B. G. Mendis, J. Mater. Chem., 2012, 22 , 11166

6. S. Stehlik, T. Petit , H. A. Girard, J. C. Arnault, A. Kromka and B. Rezek, Langmuir, 2013, $29,1634-1641$.

7. M. I. Landstrass and K. V. Ravi , Appl. Phys. Lett., 1989, 55, 975.

8. F. Maier, M. Riedel, B. Mantel, J. Ristein and L. Ley, Phys. Rev. Lett., 2000, 85 , $3472-3475$

9. V. Chakrapani, J. C. Angus, A. B. Anderson, S. D. Wolter, B. R. Stoner and G. U. Sumanasekera, Science, 2007, 318, $1424-1430$.

10. W. Yang, O. Auciello, J. E. Butler, W. Cai, J. A. Carlisle, J. E. Gerbi, D. M. Gruen, T. Knickerbocker, T. L. Lasseter, J. N. Russell, L. M. Smith and R. J. Hamers, Nat. Mater., 2002, 1, 253 -257.

11. A. Härtl , E. Schmich , J. A. Garrido, J. Hernando , S. C. R. Catharino , S. Walter , P. Feulner, A. Kromka, D. Steinmüller and M. Stutzmann , Nat. Mater., 2004, 3 , 736 742.

12. H. A. Girard, J. C. Arnault, S. Perruchas, S. Saada, T. Gacoin , J.-P. Boilot and P. Bergonzo , Diamond Relat. Mater., 2010, 19 , 1117 -1123.

13. J. C. Arnault, T. Petit, H. Girard, A. Chavanne, C. Gesset, M. Sennour and M. Chaigneau, Phys. Chem. Chem. Phys., 2011, 13, $11481-11487$.

14. H. A. Girard, T. Petit, S. Perruchas, T. Gacoin, C. Gesset, J. C. Arnault and P. Bergonzo, Phys. Chem. Chem. Phys., 2011, 13, $11517-11523$.

15. S. Q. Lud, M. Steenackers, R. Jordan, P. Bruno, D. M. Gruen, P. Feulner, J. A. Garrido and M. Stutzmann, J. Am. Chem. Soc., 2006, 128, 16884 -16891.

16. L. Ostrovskaya, V. Perevertailo, V. Ralchenko, A. Dementjev and O. Loginova, Diamond Relat. Mater., 2002,11, 845 -850.

17. A. Trouvé, I. Batonneau-Gener, S. Valange, M. Bonne and S. Mignard, J. Hazard. Mater., 2012, 201-202, 107 -114.

18. R. Akhvlediani, S. Michaelson and A. Hoffman, Surf. Sci., 2010, 604 , 2129 -2138.

19. D. Ballutaud, N. Simon, H. Girard, E. Rzepka and B. Bouchet-Fabre, Diamond Relat. Mater., 2006, 15,716 -719.

20. M. T. Edmonds, C. I. Pakes, S. Mammadov, W. Zhang, A. Tadich, J. Ristein and L. Ley, Phys. Status Solidi A, 2011, 208 , 2062 -2066.

21. D. Qi , W. Chen, X. Gao, L. Wang, S. Chen, K. P. Loh and A. T. S. Wee, J. Am. Chem. Soc., 2007, $129,8084-8085$.

22. T. Petit, J. C. Arnault, H. A. Girard, M. Sennour, T.-Y. Kang, C.-L. Cheng and P. Bergonzo , Nanoscale, 2012,4, 6792 -6799.

23. T. Petit, J. C. Arnault, H. A. Girard, M. Sennour and P. Bergonzo, Phys. Rev. B: Condens. Matter Mater. Phys., 2011, 84 , 233407.

24. E. Vanhove, J. de Sanoit, J. C. Arnault, S. Saada, C. Mer, P. Mailley, P. Bergonzo and M. Nesladek , Phys. Status Solidi A, 2007, 204 , 2931 -2939.

25. A. V. Delgado, F. González-Caballero, R. J. Hunter, L. K. Koopal and J. Lyklema, Pure Appl. Chem., 2005, 77, 1753 -1805. 
26. E. Allahyarov , I. D'Amico and H. Löwen, Phys. Rev. Lett., 1998, 81, $1334-1337$.

27. L. Lai and A. S. Barnard, J. Phys. Chem. Lett., 2012, 3, 896 -901.

28. L.-Y. Chang , E. Osawa and A. S. Barnard, Nanoscale, 2011, 3 , 958 -962.

29. S. Turner, O. I. Lebedev, O. Shenderova, I. I. Vlasov, J. Verbeeck and G. Van Tendeloo , Adv. Funct. Mater., 2009, 19 , 2116 -2124.

30. W. Chen, D. Qi , X. Gao and A. T. S. Wee , Prog. Surf. Sci., 2009, 84,279 -321.

31. C.-S. Guo, L.-B. Luo, G.-D. Yuan , X.-B. Yang, R.-Q. Zhang, W.-J. Zhang and S.-T. Lee , Angew. Chem., Int. Ed., 2009, 48 , 9896 -9900.

32. J. Asbury, E. Hao and Y. Wang , J. Phys. Chem. B, 2001, 105 , $4545-4557$.

33. O. Boussif, F. Lezoualc'h, M. A. Zanta, M. D. Mergny, D. Scherman, B. Demeneix and J. P. Behr, Proc. Natl. Acad. Sci. U. S. A., 1995, 92 , $7297-7301$ 